\title{
e-Migrinter
}

$18 \mid 2019$

L'ethnographie en migration(s)

\section{La territorialisation de la mobilisation politique de la diaspora palestinienne en Suède}

Thèse soutenue le 20 décembre 2017 à l'Université de Poitiers

\section{Fanny Christou}

\section{OpenEdition}

\section{Journals}

\section{Édition électronique}

URL : https://journals.openedition.org/e-migrinter/1593

DOI : 10.4000/e-migrinter.1593

ISSN : 1961-9685

\section{Éditeur}

UMR 7301 - Migrinter

\section{Référence électronique}

Fanny Christou, «La territorialisation de la mobilisation politique de la diaspora palestinienne en Suède », e-Migrinter [En ligne], 18 | 2019, mis en ligne le 11 septembre 2019, consulté le 20 mai 2021. URL : http://journals.openedition.org/e-migrinter/1593; DOI : https://doi.org/10.4000/e-migrinter. 1593

Ce document a été généré automatiquement le 20 mai 2021

Tous droits réservés 


\section{La territorialisation de la mobilisation politique de la diaspora palestinienne en Suède}

Thèse soutenue le 20 décembre 2017 à l'Université de Poitiers

\section{Fanny Christou}

\section{RÉFÉRENCE}

Christou, Fanny (2017) La territorialisation de la mobilisation politique de la diaspora palestinienne en Suède, Poitiers, Université de Poitiers.

\section{Questionnement à l'origine du sujet}

1 Cette thèse repose sur la volonté de mieux comprendre ce que l'expression "être Palestinien aujourd'hui » signifie, au regard des nombreuses dynamiques migratoires qui y sont liées. Même si la migration palestinienne a existé bien avant 1948, c'est à partir de cette date que l'exode de plusieurs milliers de Palestiniens a contribué à créer une diaspora de réfugiés fondée sur l'existence d'une mémoire collective (Hammer, 2005).

2 J'ai toutefois souhaité relever l'existence d'un paradoxe. Si les diasporas contemporaines sans État sont souvent associées à la recherche d'un éventuel retour et d'un sentiment d'appartenance commune (Chaliand et Rageau, 1991), le cas de la diaspora palestinienne révèle des dynamiques plus complexes. Ce sont ces dernières que j'ai voulu saisir au regard de pratiques nouvelles et de la territorialisation de la mobilisation politique au sein d'espaces variés. 


\section{Cheminement de la réflexion}

3 En partant de l'hypothèse selon laquelle le facteur politique est intrinsèquement lié à l'existence de la diaspora palestinienne, nous ne pouvons ignorer la présence du croisement des temporalités et des contextes migratoires palestiniens. Ces mutations ont généré une nouvelle structuration de la mobilité palestinienne qui repose sur l'existence de nombreux flux migratoires palestiniens au Moyen-Orient (KodmaniDarwish, 1997 ; Al-Husseini, 2011) mais aussi en Europe (Shiblak, 2005 ; Doraï, 2004). Ainsi, une recomposition de l'exil palestinien apparaît, contribuant à l'essor de reconfigurations géopolitiques et socio-spatiales de la mobilisation en diaspora.

4 Ce sont ces reconfigurations que j'ai souhaitées interroger en m'appuyant sur une période clé, celle des Accords d'Oslo. Ces derniers ont marqué l'essor de ruptures spatiales et identitaires chez les Palestiniens en raison de l'absence d'horizon politique qui y est liée. J'ai donc voulu comprendre les dynamiques spécifiques de la reterritorialisation de la mobilisation politique palestinienne au sein de l'espace suédois, territoire d'accueil situé aux marges des premiers lieux d'exil.

\section{Méthodologie et stratégies de recherche}

5 Ce travail est né d'une réflexion à partir de la ville de Malmö en Suède dans le but de comprendre ce que la diversité des espaces de la migration palestinienne recouvre en termes de mobilisation. Par une approche inductive, j'ai construit mon raisonnement en accordant la primauté à l'enquête et aux expériences quotidiennes de terrain, tout en tenant compte de l'état de l'art en lien avec mon sujet.

6 L'important matériel recueilli sur le terrain n'a pu être analysé que par le choix de rester en Suède pour le travail de mise en mots. J'ai ainsi voulu saisir les évolutions, ruptures et moments clés de la mobilisation palestinienne à Malmö, pour appréhender au mieux la réalité vécue par les Palestiniens en Suède.

\section{Méthodologie d'investigation}

7 J'ai réalisé 46 entretiens semi-directifs auprès de membres de la diaspora palestinienne nés en Jordanie, au Liban, en Syrie ou encore dans les Territoires palestiniens occupés. Les thématiques abordées sont en lien avec le parcours migratoire, les activités militantes dans le pays d'origine ainsi qu'en Suède (héritées ou construites), le rapport à l'identité et les processus d'intégration au sein des différents espaces. J'ai aussi effectué 24 entretiens informels dans le but de comprendre la manière dont les formes de mobilisation varient selon les individus.

En complément, j'ai réalisé plus de 30 observations participantes au sein d'organisations palestiniennes basées à Malmö (associations politiques et culturelles) mais aussi lors de manifestations et divers évènements. Par mon immersion constante, ce travail de terrain ethnographique permet de comprendre les ressorts des dynamiques de mobilisation palestinienne. Les observations "flottantes " menées au sein de la société suédoise et parmi les membres la diaspora palestinienne ont mis en lumière les espaces fréquentés, les pratiques socio-politiques, les manières d'être au quotidien. 


\section{Méthodologie d'analyse}

Ces investigations de terrain ont laissé place à un travail d'analyse par une approche thématique transversale. Cette dernière a révélé l'importance de deux facteurs autour de deux niveaux d'analyse complémentaires pour interroger la territorialisation de la mobilisation politique palestinienne en Suède: les trajectoires migratoires et les parcours militants.

\section{Problématique}

10 J'ai alors construit ma problématique en cherchant à comprendre ce que le croisement des trajectoires migratoires et militantes signifie en termes de pratiques. Ainsi : Que révèle l'analyse des trajectoires migratoires et militantes des Palestiniens en Suède sur les reconfigurations territoriales et politiques de la diaspora palestinienne en Suède depuis les Accords d'Oslo?

11 L'originalité de ma thèse repose sur l'analyse des pratiques de mobilisation de la diaspora palestinienne, par la mise en lumière de l'existence d'une territorialisation du politique spécifique. Au terme de l'exploitation croisée de ces ressources, j'ai pris en compte trois dimensions : celle des espaces, des acteurs et des outils. Les trois parties transversales de cette thèse sont liées par un même fil conducteur: celui de la mobilisation politique en définissant où, qui et comment ce processus peut être redéfini à la lumière du cas palestinien.

\section{Principaux résultats et apports significatifs}

\section{Le rôle de la Suède et de l'espace local dans l'accueil des réfugiés palestiniens}

12 Cette thèse donne à voir le rôle d'un pays où la présence numérique palestinienne peut s'avérer à première vue marginale. Nos enquêtes de terrain auprès de l'Agence des migrations, du Bureau des statistiques suédoises et de groupes locaux permettent d'estimer le nombre de Palestiniens en Suède à 30000 en 2016, dont 5000 dans la ville de Malmö.

13 Au fil de l'histoire, la question palestinienne a investi l'agenda politique suédois, avec un certain soutien à la cause des migrants ainsi qu'à celle des Palestiniens de la part des partis politiques de gauche. Constituant un « centre de gravité » (Khayati, 2012) pour de nombreuses communautés, « le multiculturalisme et l'État providence suédois ont servi de modèle à de nombreux États » (Eliassi, 2014 : 145). Ainsi, les communautés kurdes (Eliassi, 2014 ; Khayati, 2012) ou chiliennes (Olsson, 2009 ; Sznajder et Ronigher, 2007) par exemple, ayant cherché refuge en Suède, souhaitent trouver dans l'action collective un moyen de se mobiliser pour faire entendre leur cause. Les Palestiniens ont à cet égard recours aux structures d'opportunité politique de ce pays, doublées par une politique migratoire longtemps considérée comme généreuse même si les derniers mois de notre terrain ont révélé certaines nuances.

14 Ainsi, un cadre de compréhension quant au lien existant entre développement de la mobilisation palestinienne en diaspora et espace local suédois, a été apporté. Le 
contexte local suédois donne un sens particulier aux pratiques palestiniennes à Malmö, mais comment expliquer les différentes formes de mobilisation? L'analyse de dynamiques à deux niveaux a permis une réinterprétation de la mobilisation palestinienne en diaspora.

\section{Être Palestinien en Suède : les dynamiques au niveau exogène}

En effet, au niveau exogène, j'ai souhaité réinterpréter les dynamiques relatives aux pratiques de mobilisation en exil, apportant une relecture de la réalité socio-politique de la mobilité palestinienne. Aussi, j'ai voulu rendre compte de l'importance de la prise en compte de deux facteurs principaux qui participent à préciser, dans le champ d'étude des diasporas, ce que la mobilisation palestinienne en Suède traduit, à savoir les fragmentations migratoires / spatiales et les fragmentations politiques.

Tout d'abord, les parcours migratoires palestiniens témoignent d'enjeux différents de mobilisation en raison des fragmentations qui y sont rattachées. Ces parcours reposent sur trois facteurs : la cause de la migration, le contexte et la période d'arrivée en Suède. Combinées à ces parcours migratoires, les fragmentations spatiales, entendues comme les différenciations d'intégration socio-politique au sein de différents lieux d'accueil, contribuent, elles aussi, à reconfigurer les pratiques de mobilisation. La complexité des parcours migratoires palestiniens installe alors le processus de leur mobilité dans une forme de « provisoire qui dure».

De ces fragmentations migratoires et spatiales, apparaissent transversalement des fragmentations d'ordre politique que les Accords d'Oslo ont renforcé parmi les membres de la diaspora palestinienne. En fonction des lieux, la politisation de la question palestinienne a contribué à territorialiser la mobilisation politique au sein d'espaces qui sont devenus le creuset de la résistance palestinienne en exil. Aussi, les parcours militants des Palestiniens permettent le renforcement de capitaux alors réinvestis au sein de nouveaux lieux d'exil.

\section{Être Palestinien en Suède : les dynamiques au niveau endogène}

Au niveau endogène, ces espaces variés d'exil, au sein desquels se sont développées des formes diverses de mobilisation, ont parallèlement engendré un renforcement des fragmentations identitaires contribuant à une réinterprétation de l'être palestinien aujourd'hui. Ces cristallisations justifient l'apparition d'une reconfiguration des pratiques mais aussi des appartenances, pouvant être interprétées à une double échelle.

En effet, les Palestiniens manifestent une capacité d'action collective par la mise en place de pratiques quotidiennes. Mais en articulant les processus identitaires et d'intégration aux notions de capitaux socio-politiques et mobilitaires (Kaufmann et al., 2004 ; Ceriani Sebregondi, 2007) tout autant que de socialisation anticipée (Merton, 1957), j'ai souhaité relire les pratiques de la diaspora palestinienne, sous un angle individuel.

\section{Le croisement des niveaux exogène et endogène}

Ainsi, le croisement des niveaux exogène et endogène permet une réinterprétation de la mobilisation palestinienne par (1) l'articulation et la transversalité des pratiques 
individuelles et collectives, (2) la diversité des parcours migratoires (causes, contextes et temporalités) et militants (capitaux, socialisations et héritages), (3) la pluralité des référents identitaires et des rapports à l'intégration. Ces liens de cause à effet peuvent être illustrés comme suit.

Figure $n^{\circ} 1$ : Tableau retraçant les principaux résultats obtenus

\begin{tabular}{|c|c|c|}
\hline $\begin{array}{l}\text { Vagues migratoires } \\
\text { palestiniennes en } \\
\text { Suède }\end{array}$ & Mobilisation en Suède & Principaux facteurs d'explication \\
\hline $\begin{array}{l}\text { Premières vagues } \\
\text { migratoires de la } \\
\text { Jordanie et du Liban }\end{array}$ & $\begin{array}{l}\text { Mobilisation communicationnelle } \\
\text { rationnelle: recherche d'une } \\
\text { valorisation personnelle, soutien à la } \\
\text { cause palestinienne et volonté } \\
\text { d'intégration en Suède }\end{array}$ & $\begin{array}{l}\text { Militantisme traditionnel dans le } \\
\text { pays d'origine au sein duquel il } \\
\text { existait une forte intégration } \\
\text { politique mais des conditions de } \\
\text { vie difficiles }\end{array}$ \\
\hline $\begin{array}{l}\text { Vagues migratoires } \\
\text { récentes de Syrie }\end{array}$ & $\begin{array}{l}\text { Mobilisation connectée } \\
\text { silencieuse ancrée } \\
\text { syrienne }\end{array}$ & $\begin{array}{l}\text { Activités militantes dans le pays } \\
\text { d'origine au sein duquel il } \\
\text { existait une faible intégration } \\
\text { politique mais des conditions de } \\
\text { vie socio-économique plus } \\
\text { favorables }\end{array}$ \\
\hline $\begin{array}{l}\text { Vagues migratoires } \\
\text { anciennes et récentes } \\
\text { des territoires occupés } \\
\text { palestiniens }\end{array}$ & $\begin{array}{l}\text { Mobilisation } \quad \text { socio-culturelle } \\
\text { silencieuse }\end{array}$ & $\begin{array}{l}\text { Activités militantes dans le pays } \\
\text { d'origine marquées par une } \\
\text { absence d'horizon politique et } \\
\text { une déception des structures } \\
\text { politiques }\end{array}$ \\
\hline
\end{tabular}

Source : Christou, 2017.

\section{Conclusion}

L'aspect novateur de ce travail repose sur la caractérisation de l'originalité de la réalité socio-politique de la diaspora palestinienne. Les principales contributions de ce travail reposent sur l'analyse tant spatiale et que socio-politique des phénomènes de mobilisation, prenant en compte l'intersection de plusieurs variables et de diverses échelles, permettant d'éclairer les pratiques d'un groupe étudié par les marges.

travail de thèse permet ainsi de rendre compte de limportance de la territorialisation du facteur politique en Suède qui est constitutif de la réalité sociopolitique de la diaspora palestinienne. L'originalité de la mobilisation palestinienne en diaspora peut alors être définie comme une forme d'engagement transnational: les activités militantes n'ont pas disparu mais peuvent être réinterprétées au regard des mutations géopolitiques (tant migratoires / spatiales que militantes / politiques).

L'analyse des liens entre espaces de la migration palestinienne et pratiques de mobilisation en diaspora permet ainsi de saisir la réalité socio-politique des dynamiques de la mobilité palestinienne. Par une analyse novatrice micro-située, cette 
recherche s'est ainsi plongée dans l'espace suédois de mobilisation palestinienne pour comprendre l'interaction de plusieurs niveaux d'analyse. Les trajectoires migratoires et les parcours militants des Palestiniens révèlent ainsi une territorialisation spécifique de leur mobilisation dans un lieu en marge des espaces de premier exil au MoyenOrient. J'ai pu alors saisir l'importance de ces deux facteurs dans l'articulation originale $\mathrm{du}$ renforcement d'une identité collective en diaspora et du développement de représentations individuelles, alors ancrée au sein d'un espace transnational. En donnant directement la parole aux membres de la diaspora palestinienne en Suède, j'ai donc voulu appréhender sa réalité socio-politique construite à différents niveaux. Les témoignages recueillis traduisent ainsi l'importance du lien entre le faire politique et l'action d'être palestinien malgré les diverses expériences d'exil.

\section{BIBLIOGRAPHIE}

Al-Husseini, Jalal ; Signoles, Aude (2011) Les Palestiniens entre État et diaspora. Le temps des incertitudes, Paris, IISMM / Karthala, 456 p.

Ceriani Sebregondi, Giorgia (2007) La circulation migratoire aujourd'hui : Émergence d'un capital mobilitaire et d'une culture de la mobilité. Le cas des migrants marocains en Europe du Sud, in Audebert, C. ; Ma Mung, E.(dir.) Les nouveaux territoires migratoires : entre logiques globales et dynamiques locales, Bilbao, Université de Deusto, pp. 243-254.

Chaliand, Gérard ; Rageau, Jean-Pierre (1991) Atlas des diasporas, Paris, Odile Jacob, 183 p.

Doraï, Mohamed Kamel (2004) Les réfugiés palestiniens en Europe. Complexité des parcours et des espaces migratoires, Revue Européenne des Migrations Internationales, vol. 20, nº 2, pp. 169-186.

Eliassi, Barzoo (2014) La diaspora kurde en Suède, Hommes et migrations, $\mathrm{n}^{\circ}$ 1307, pp. 144-149.

Kaufmann, Vincent ; Bergman, Manfred Max ; Joye, Dominique (2004) Motility : mobility as capital, International Journal of Urban and Regional Research, vol. 28, in ${ }^{\circ} 4$, pp. 745-756.

Khayati, Khalid (2012) Sweden as a gravitation center for the Kurds - Diaspora formation and transnational relations, in Heshmati, A. ; Dilani, A. ; Baban, S.M.J. (eds) Perspectives on Kurdistan's Economy and Society in Transition, New-York, Nova Science Publisher Inc., pp. 101-114

Kodmani-Darwish, Bassma (1997) La diaspora palestinienne, Paris, Presses universitaires de France, $264 \mathrm{p}$.

Merton, Robert K. (1957) Éléments de théorie et de méthode sociologique, Paris, Armand Colin / Masson, $384 \mathrm{p}$.

Olsson, Erik (2009) From exile to post-exile : the diasporisation of Swedish Chileans in historical contexts, Journal for the Study of Race, Nation and Culture, vol. 15, n 5, pp. 659-676.

Shiblak, Abbas (2005) Palestinian refugees in Europe: Challenges of adaptation and identity, Summary Report on a Workshop on Palestinian Refugee Communities in Europe, St. Anthony's College ; University of Oxford. 
Sznajder, Mario ; Ronigher, Luis (2007) Exile Communities and Their Differential Institutional Dynamics : A Comparative Analysis of the Chilean and Uruguayan Political Diasporas, Revista de Ciencia Politica, vol. 27, $\mathrm{n}^{\circ}$ 1, pp. 43-66.

\section{RÉSUMÉS}

Ce texte retrace la manière dont j'ai présenté mes travaux lors de ma soutenance, par la prise en compte de facteurs multidimensionnels, à la croisée de contextes et de temporalités variés. Les principaux résultats mettent l'accent sur la mobilité palestinienne au miroir de l'évolution des formes de mobilisation en Suède. Par l'analyse des pratiques socio-politiques des Palestiniens en Suède, cette thèse contribue à relire le sens politique donné à la territorialisation de la mobilisation palestinienne dans le contexte post-Oslo.

\section{INDEX}

Mots-clés : diaspora, mobilisation politique, territorialisation, Palestinien Index géographique : Suède, Moyen-Orient

\section{AUTEURS}

\section{FANNY CHRISTOU}

Docteure en Géographie, Migrinter (CNRS / Université de Poitiers), Post-doctorante à la Fondation Croix Rouge Française et chercheuse au Center for Middle Eastern Studies, Lund University

fanny.christou@gmail.com 